\title{
Induction of Trehalase in Arabidopsis Plants Infected With the Trehalose-Producing Pathogen Plasmodiophora brassicae
}

\author{
David Brodmann, ${ }^{1}$ Astrid Schuller, ${ }^{2}$ Jutta Ludwig-Müller, ${ }^{2}$ Roger A. Aeschbacher, ${ }^{1}$ Andres Wiemken, \\ Thomas Boller, ${ }^{1}$ and Astrid Wingler ${ }^{1}$ \\ ${ }^{1}$ Botanisches Institut, Universität Basel, Hebelstrasse 1, CH-4056 Basel, Switzerland; ${ }^{2}$ Institut für Botanik, Technische \\ Universität Dresden, D-01062 Dresden, Germany.
}

Submitted 31 December 2001. Accepted 16 March 2002.

\begin{abstract}
Various microorganisms produce the disaccharide trehalose during their symbiotic and pathogenic interactions with plants. Trehalose has strong effects on plant metabolism and growth; therefore, we became interested to study its possible role in the interaction of Arabidopsis thaliana with Plasmodiophora brassicae, the causal agent of clubroot disease. We found that trehalose accumulated strongly in the infected organs (i.e., the roots and hypocotyls) and, to a lesser extent, in the leaves and stems of infected plants. This accumulation pattern of trehalose correlated with the expression of a putative trehalose-6-phosphate synthase (EC 2.4.1.15) gene from $P$. brassicae, PbTPS1. Clubroot formation also resulted in an induction of the Arabidopsis trehalase gene, $A T T R E 1$, and in a concomitant increase in trehalase (EC 3.2.1.28) activity in the roots and hypocotyls, but not in the leaves and stems of infected plants. Thus, induction of $A T T R E 1$ expression was probably responsible for the increased trehalase activity. Trehalase activity increased before trehalose accumulated; therefore, it is unlikely that trehalase was induced by its substrate. The induction of trehalase may be part of the plant's defense response and may prevent excess accumulation of trehalose in the plant cells, where it could interfere with the regulation of carbon metabolism.
\end{abstract}

Additional keywords: ADP-glucose pyrophosphorylase, auxin, cytokinin.

The obligate biotroph Plasmodiophora brassicae is the causal agent of clubroot in the family Brassicaceae, a disease characterized by stunted growth and by gall formation in the root system of infected plants (Ludwig-Müller 1999). Some lower organisms, among them $P$. brassicae, often previously referred to as "lower fungi", are now considered to belong either to the kingdom of Protozoa (Agrios 1997) or Prot(oct)ista (Margulis et al. 1989). In the latter, groups of closely related organisms are found that do not fit into the classic fungus/plant/animal classifications. $P$. brassicae is placed in the Plasmodiophoromycetes class within the phylum Plasmodio-

Corresponding author: Astrid Wingler; Department of Biology, University College London, Gower Street, London WC1E 6BT, U.K.; E-mail: a.wingler@ucl.ac.uk. Fax: +442076797096.

The nucleotide sequence data have been submitted to GenBank and are available under accession numbers AF334707 for the genomic sequence of PbTPS1 and AF334708 for the cDNA sequence of PbTPS1. phoromycota. Together with other plant pathogens of the genus Polymyxa and Spongospora, Plasmodiophora composes the order of Plasmodiophorales (Agrios 1997).

The life cycle of this obligate pathogen consists of two phases: the primary phase, which is restricted to root hairs of the infected plant, and the secondary phase, which results in the colonization of the cortex and stele of the hypocotyls and roots (Ingram and Tommerup 1972). The clubroot symptoms clearly give evidence that plant growth regulators are involved in disease development. Hyperplasia and hypertrophy indicate the involvement of auxins (Ludwig-Müller et al. 1993) and cytokinins (Dekhuijzen and Overeem 1971). Although cytokinins are most likely synthesized by plasmodia of the pathogen (Dekhuijzen 1981; Müller and Hilgenberg 1986), it appears that the disease stimulates production of the auxin indole 3acetic acid in the host (Grsic et al. 1999; Grsic-Rausch et al. 2000).

Other symptoms of clubroot disease include changes in carbohydrate metabolism, such as an accumulation of starch in the roots and hypocotyls of infected plants (Evans and Scholes 1995; Williams et al. 1968). In addition, the disaccharide trehalose ( $\alpha$-D-glucopyranosyl-[1,1]- $\alpha$-D-glucopyranoside) has been shown to accumulate in the hypocotyls of cabbage plants affected by clubroot disease (Keen and Williams 1969). Trehalose, which is usually synthesized by trehalose-6-phosphate synthase (TPS) and trehalose-6-phosphate phosphatase, is a typical microbial sugar accumulating in a variety of symbiotic or pathogenic interactions of microorganisms with plants (Müller et al. 1995a). The synthesis of trehalose could affect the carbon metabolism of infected plants in two ways: By converting plant photosynthates into trehalose, microorganisms would divert carbon away from plant metabolism and thereby create a carbon sink. In addition, trehalose that is released into the plant could affect plant metabolism by interfering with the plant's sugar sensing system. For example, trehalose has been shown to induce enzymes of fructan synthesis in barley (Müller et al. 2000; Wagner et al. 1986) and sucrose synthase activity in soybean (Müller et al. 1998). In Arabidopsis thaliana, growth on trehalose-containing medium results in an inhibition of root growth, an increased accumulation of starch in the shoots, and an increased activity of ADP-glucose pyrophosphorylase, the first enzyme of starch synthesis, accompanied by an induction of the ADP-glucose pyrophosphorylase gene ApL3 (Fritzius et al. 2001; Wingler et al. 2000).

Plants such as A. thaliana are themselves capable of synthesizing small amounts of trehalose (Blázquez et al. 1998; Müller et al. 2001; Vogel et al. 1998, 2001); therefore, treha 

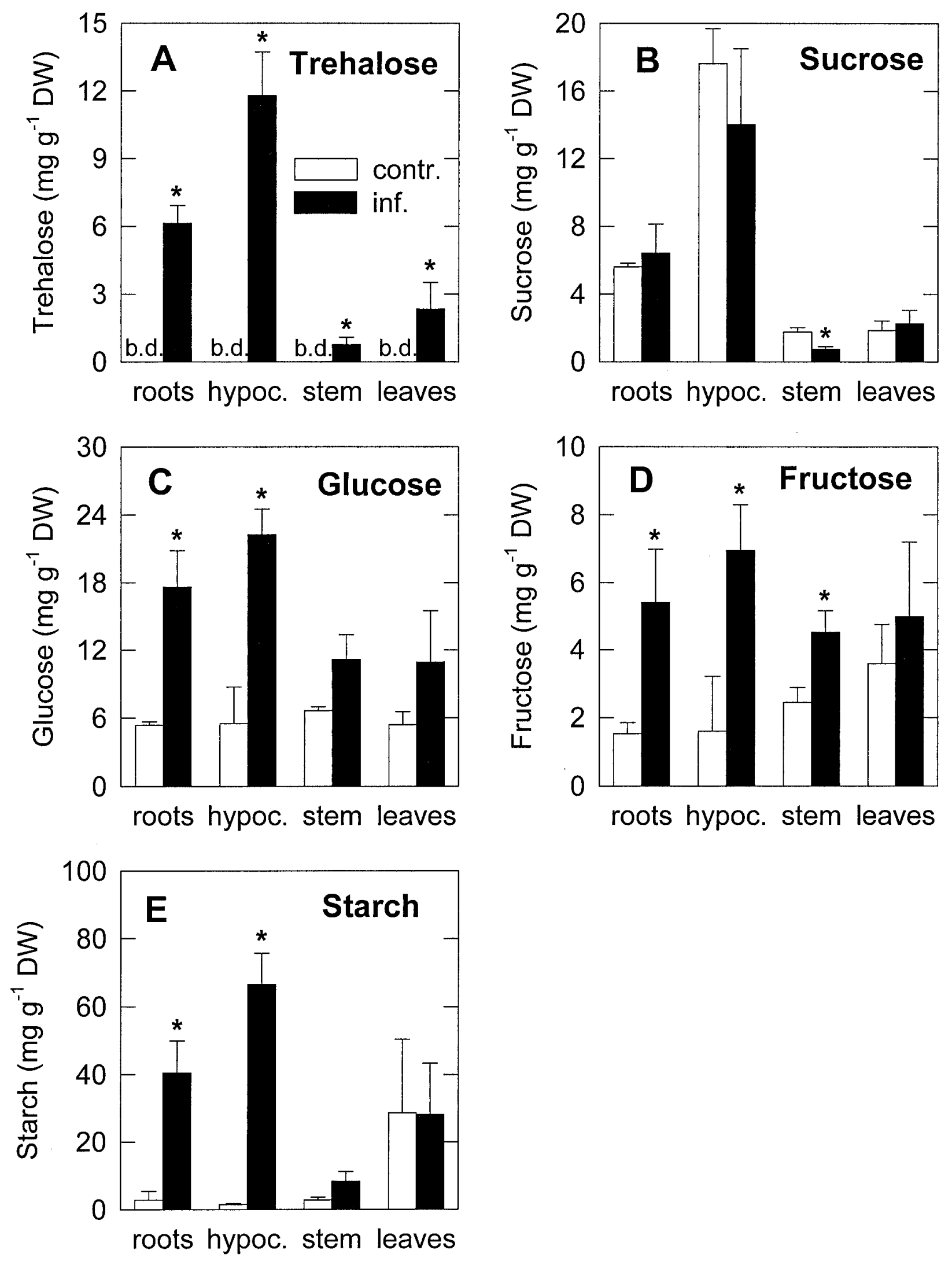

Fig. 1. Amount of A, trehalose, B, sucrose, $\mathbf{C}$, glucose, D, fructose, and $\mathbf{E}$, starch in the roots, hypocotyls, stems, and cauline leaves of control plants (control $=$ open bars) and of infected plants (infected = black bars) 38 days after inoculation with Plasmodiophora brassicae. Data are means of between four and five plants \pm standard error. Asterisks $(*)$ indicate significant differences between the control and the infected plants $($ analysis of variance; $P \leq 0.05)$. b.d. $=$ below detection limit. 
lose synthesis also may be an important intrinsic component in the regulation of plant metabolism (Goddijn and Smeekens 1998; Müller et al. 1999). In fact, an Arabidopsis mutant with a T-DNA insertion disrupting AtTPS1 is embryo lethal, indicating that trehalose synthesis plays an essential role in plants (Eastmond et al. 2002). By releasing trehalose into the plant, pathogens such as $P$. brassicae might be able to exploit the plant's trehalose-sensing system and alter carbohydrate metabolism to their favor.

The concentration of trehalose in plant cells is probably regulated by trehalase, the enzyme catalyzing the hydrolysis of trehalose into two glucose molecules. By preventing an overaccumulation of trehalose that would interfere with the regulation of plant metabolism, trehalase could play an important role in plant-microbe interactions. For example, trehalase activity has been found to be increased in symbiotic relationships of plants with trehalose-producing microorganisms, such as arbuscular mycorrhizal fungi (Schubert and Wyss 1995) and rhizobia (Müller et al. 1994).

Here, we studied the possible role of trehalose metabolism in the interaction of $A$. thaliana with $P$. brassicae, focusing on the amounts of trehalose accumulating in various plant organs and on the activity and expression of plant trehalase.

\section{RESULTS}

Effect of clubroot formation on carbohydrate metabolism.

Thirty-eight days after inoculation with $P$. brassicae, each of the infected Arabidopsis plants clearly showed gall formation, the typical symptom of clubroot disease. At this stage, the pools of soluble sugars and starch were determined in various organs of the plants (Fig. 1). Trehalose, which was below the detection limit in healthy plants, became one of the predominant soluble sugars in the roots and hypocotyls of infected plants (Fig. 1A). Smaller amounts of trehalose were also found in the cauline leaves and in the stems of infected plants. No trehalose was detectable in infected plants harvested 14 and 23 days after inoculation, indicating that trehalose is formed only during the later stages of clubroot development. Apart from a reduction in the sucrose content in the stems, clubroot formation had no significant effect on sucrose formation (Fig. 1B). In contrast, glucose (Fig. 1C) and fructose (Fig. 1D) contents were generally increased in the infected plants. This effect was most pronounced in the roots and hypocotyls, where the hexose contents increased more than threefold. In addition, infection with $P$. brassicae led to a 14- and 45-fold increase in the starch content in the roots and hypocotyls, respectively (Fig. 1E). In the stems, starch accumulated to a smaller extent, while there was no effect of infection with $P$. brassicae on the starch content in the cauline leaves. However, starch accumulated in the rosette leaves of infected plants at earlier stages of clubroot development (data not shown).

To study if the accumulation of starch in the roots and hypocotyls was due to an induction of gene expression, we analyzed the expression of the Arabidopsis ADP-glucose pyrophosphorylase genes, $A p L 1, A p L 2, A p L 3$, and $A p S$. As previously shown, exogenously supplied trehalose leads to an induced expression of $A p L 3$ and causes starch accumulation in shoots of $A$. thaliana seedlings (Wingler et al. 2000). However, despite the accumulation of trehalose in the infected roots and hypocotyls, there was apparently no induction of any of the ADP-glucose pyrophosphorylase genes (Fig. 2).

\section{Isolation of a putative TPS gene fragment from $P$. brassicae and its expression.}

To test if the expression of the P. brassicae TPS correlated with the accumulation of trehalose during the clubroot disease, we cloned and sequenced a fragment of a $P$. brassicae gene (PbTPS1) showing homology to known TPS genes. P. brassi$c a e$ is an obligate biotroph and cannot be grown in pure culture; therefore, we used cDNA from infected Arabidopsis roots and degenerate primers for fungal TPS genes in an attempt to amplify a respective DNA fragment by polymerase chain reaction (PCR). We indeed obtained a cDNA fragment, PbTPS1, with a size of $302 \mathrm{bp}$. After removing the original primer sequences and translation into a protein sequence of 86 amino acids, database searches were carried out to confirm that PbPTS1 encodes a TPS protein. Search of the ProDom protein domain database (INRA, Toulouse, France) identified PbTPS1 as member of the TPS protein family. Additional blast searches revealed that the predicted protein sequence shows high homology to fungal TPS proteins (Fig. 3A), especially to the tpsA protein from Aspergillus nidulans (59\% identity). In addition, the PbTPS1 sequence also is homologous to TPS proteins of plant origin (55 and $51 \%$ identity to the Arabidopsis AtTPS1 and the Selaginella lepidophylla SITPS1 proteins, respectively). To confirm that the PbTPS1 fragment originated from $P$. brassicae, we designed specific primers based on the nucleotide sequence of the isolated fragment and used these primers to amplify a corresponding DNA fragment using genomic DNA from resting spores of $P$. brassicae. With this approach, we obtained a fragment that, apart from containing an intron, was identical to the fragment isolated using the cDNA from infected roots (Fig. 3B), confirming that the PbTPS1 fragment originated from $P$. brassicae.

To analyze if the expression of PbTPS1 correlated with the ability of $P$. brassicae to form trehalose, we determined the trehalose content and the expression of PbTPS1 in resting spores. Resting spores of $P$. brassicae contained trehalose at $14.7 \pm 1.6$ $\mathrm{mg} \mathrm{g}^{-1}$ dry weight (mean of three extracts \pm standard error). Two bands were detected in the reverse transcription (RT)-PCR analysis of PbTPS1 expression (Fig. 4, lane +DNAse +RT). Sequencing of the bands revealed that the smaller amplicon (243 bp) corresponded to the PbTPS1 cDNA, while the larger amplicon (301 bp) was identical with the genomic PbTPS1 DNA sequence (Fig. 4, lane -DNAse-RT). Neither band could be detected without reverse transcription; therefore, it is likely that the larger band represented the amplification product of cDNA from unprocessed PbTPSI transcript and was not the result of contamination with genomic DNA.

In correspondence with the occurrence of trehalose, PbTPS1 was expressed in the roots and hypocotyls of infected plants, whereas no expression of PbTPS1 was detectable in other parts of the infected plants or in control plants (Fig. 5). Expression of the Arabidopsis TPS gene, AtTPS1, in the roots and stems was unaffected by infection with $P$. brassicae.

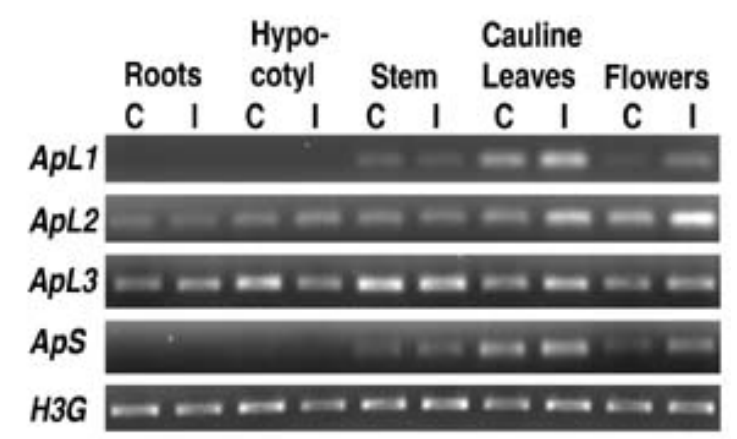

Fig. 2. Reverse-transcription polymerase chain reaction analysis of the expression of the Arabidopsis ADP-glucose pyrophosphorylase genes, $A p L 1, A p L 2, A p L 3$, and $A p S$ and of the histone gene $H 3 G$ in roots, hypocotyls, stems, and cauline leaves 38 days after inoculation with Plasmodiophora brassicae; $\mathrm{C}=$ control plants and $\mathrm{I}=$ infected plants. 
Induction of trehalase activity and of gene expression.

Clubroot formation resulted in a strong increase in trehalase activity in the roots (including the hypocotyls). This increase was already observed 14 days after inoculation and persisted during the whole experiment (Fig. 6A). Trehalase activity was, however, not affected in the stems (Fig. 6B) or in the leaves (Fig. 6C) of infected plants. We also analyzed the expression of the Arabidopsis trehalase gene (Müller et al. 2001). Expression of ATTRE1 was induced in the roots and hypocotyls of infected plants (Fig. 5), but not in the stems or leaves, indicating that the increased trehalase activity was, at least in part, due to enhanced expression of trehalase in the host plant. In the flowers, the expression of ATTREl was generally high, but not affected by clubroot formation.

\section{DISCUSSION}

Our results show that trehalose accumulates during clubroot formation in A. thaliana (Fig. 1), confirming earlier work by Keen and Williams (1969) on clubroot-diseased cabbage plants. In addition to trehalose, hexoses and starch accumulated in the roots and hypocotyls of infected plants (this study;

\section{A}

P. brassicae

A. nidulans

A. niger

$S$. pombe

Y. lipolytica

$P$. angusta

C. albicans

S. lepidophylla

A. thaliana

P. brassicae

A. nidulans

A. niger

$S$. pombe

Y. Iipolytica

$P$. angusta

C. albicans

S. Iepidophylla

A. thaliana
MLLPGILRTAS - - - - PKMQIGFFLHTPFPSSEIYRILPQRDEIVHSLL - 44

MLLPEMLREEIGNT - -KKNIKIGFFLHTPFPSSEIYRILPVRNELLLGLL - 48

MLLPEMLREEIGDS - -KENVKIGFFLHTPFPSSEIYRILPVRNELLLGVL -48

MVLPQMLRELIGDK - -FKDIKIGFFLHTPFPSSEIYRVLPVRNEILEGVL -48 MLLPEMLREECENNSALDGLKIGFFLHTPFPSSEIYRILPVRKEVLTGVL - 50 MLLPSLLRDQLNSKG-LPNVKIGFFLHTPFPSSEIYRILPVRKEILEGVL - 9 MLLPEMLRQEIGNK- -KKNIKIGFFLHTPFPSSEIYRILPVRKEILEGVL -48

MFLPSYLKEKD - - - - SQMKVGWFLHTPFPSSEIYRTLPLRAELIQGVI -44 MFLPKCLKEYN - - - - -SKMKVGWFLHTPFPSSEIHRTLPSRSELLRSVL -44 $*$ ** * : $:::^{*}: * * * * * * * * * * * * * * *::$ : :*

SSDLIGFQVYDYCRHFMTSCTRVLG-MDNPPNGGQLGKGLATV - 86 HCDLIGFHTYDYTRHFLSACSRLLG-LPTTPNGIEFQGKIIAC -90 HCDLIGFHTYDYTRHFLSACSRLLG-LTTTPNGIEFQGKIIAC -90 NCDLVGFHTYDYARHFLSACSRILN-LSTLPNGVEYNGQMVSV - 90 SCNLIGFHTYDYARHFLSSVSRILD-LETMPNGTYYKGRHVVV - 92 SCDLIGEHTYDYVRHFLSSVERILK-LRTSPQGVVYNDRQVTV - 91 SCDLIGFHTYDYARHFISSVSRIVPNVSTLPNGIKYQGRSISI - 91

AADLVGFHTYDYARHFVSACTRILG-LEGTPEGVEDQGKNTRV - 86 AADLVGFHTYDYARHFVSACTRILG-LEGTPEGVEDQGRLTRV - 86 .:*:**:.*****::: *:: : *:*

B

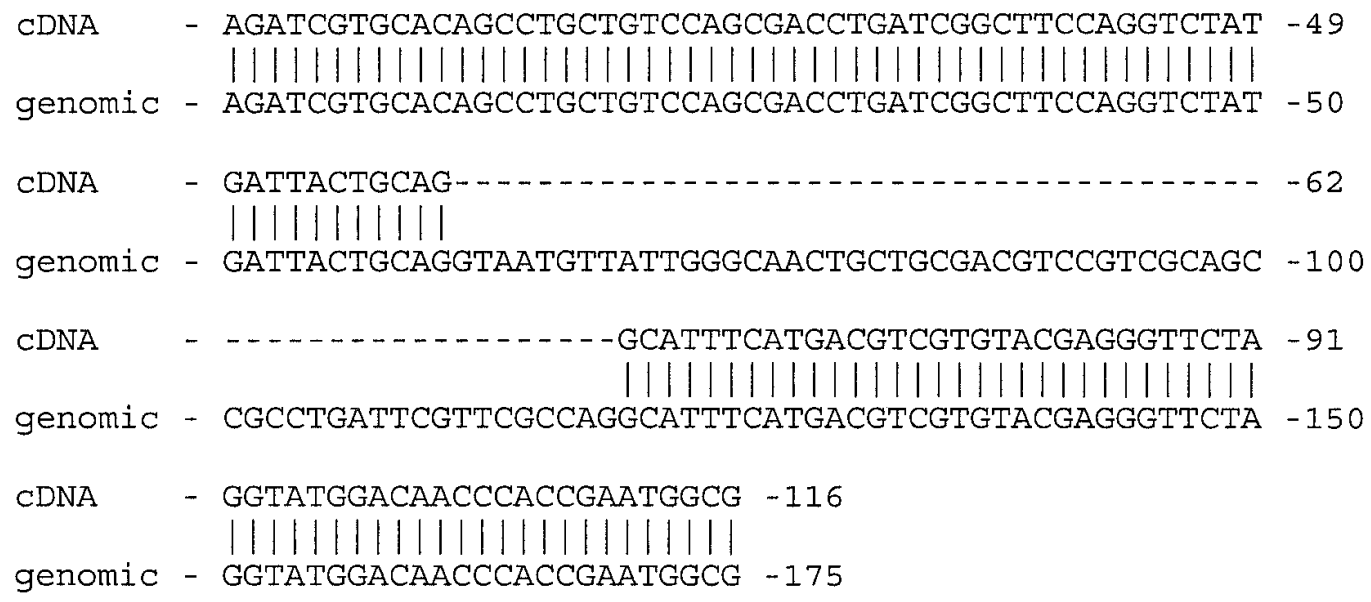

Fig. 3. A, Alignment of the predicted amino acid sequence encoded by the trehalose-6-phosphate synthase (TPS) gene fragment from Plasmodiophora brassicae with the sequences of the TPS proteins from Aspergillus nidulans (accession number AF043230), Aspergillus niger (accession number U07184), Schizosaccharomyces pombe (accession number Z29971), Yarrowia lipolytica (accession number AJ011032), Pichia angusta (accession number AJ010725), Candida albicans (accession number Y07918), Selaginella lepidophylla (accession number U96736), and Arabidopsis thaliana (accession number Y08568). Asterisks (*) indicate identical or conserved residues in all sequences, colons (:) indicate conserved substitutions, and dots (.) indicate semiconserved substitutions. B, Alignment of the nucleotide sequences obtained by isolating the PbTPS1 fragment using cDNA from infected Arabidopsis roots or genomic DNA from resting spores of $P$. brassicae. 
Evans and Scholes 1995; Williams et al. 1968), and it was suggested that the regulation of starch synthesis by the host may be under parasite control (Williams et al. 1968). Exogenously supplied trehalose can induce an overaccumulation of starch in the shoots of $A$. thaliana (Fritzius et al. 2001; Wingler et al. 2000); therefore, we became interested to analyze whether trehalose could be responsible for the accumulation of starch observed during clubroot disease. In addition, we studied the involvement of trehalase in preventing potentially deleterious effects of trehalose on the host plant.

Most microorganisms have the capacity to accumulate significant amounts of trehalose; therefore, it was likely that the accumulation of trehalose in infected plants was mainly due to trehalose synthesis by $P$. brassicae. The high trehalose content in resting spores of $P$. brassicae, exceeding the content in infected Arabidopsis tissue, confirms that $P$. brassicae is capable of synthesizing significant amounts of trehalose. Although $A$. thaliana also has the ability to form small amounts of trehalose, the trehalose content in Arabidopsis plants is usually very low, and significant amounts of trehalose are detectable only when trehalase activity is inhibited by addition of validamycin A (Müller et al. 2001; Vogel et al. 2001). Given the constitutively low expression of AtTPS1 (Fig. 5), the only Arabidopsis gene known to encode a functional TPS (Blázquez et al. 1998; Vogel et al. 2001), it is likely that the trehalose accumulating during clubroot disease was formed by $P$. brassicae and not by A. thaliana.

Due to its obligate parasitism, information on physiological processes in $P$. brassicae is very scarce. Only recently, the first sequence coding for an mRNA of $P$. brassicae induced in planta was reported (Ito et al. 1999). However, after database searches, it was difficult to assign a function to this mRNA. In contrast, the PbTPS1 gene of $P$. brassicae, which we show here to be expressed in planta, was identified as a trehalose-6-phosphate synthase gene, based on the high homology of the predicted protein sequence to other TPS proteins (Fig. 3A). In addition to the expression of PbTPS1 in infected plants, the presence of PbTPS1 transcript also corresponded with the accumulation of trehalose in resting spores of $P$. brassicae (Fig. 4). In accordance with the observation that $P$. brassicae is restricted to the roots and the hypocotyls (Mithen and Magrath 1992), and only under certain circumstances (short days, late flowering population) can be found in other host tissues such as leaves and stems (Koch et al. 1991; Mithen and Magrath 1992), expression of PbTPS1 was detected only in the roots and hypocotyls of infected Arabidopsis plants, but not in the leaves, stems, or flowers. The cloning of PbTPS1 makes it possible to use PbTPS1 expres-

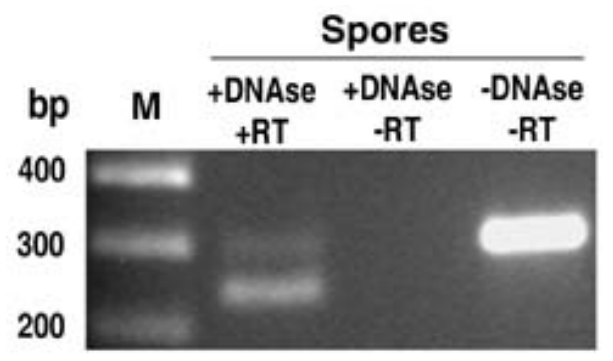

Fig. 4. Reverse transcription-polymerase chain reaction analysis of the expression of PbTPS1 in resting spores of Plasmodiophora brassicae. The smaller amplicon (243 bp) corresponds to the PbTPS1 cDNA sequence, and the larger amplicon (301 bp) corresponds to the genomic DNA sequence. $\mathrm{M}=$ marker; +DNAse + RT = treatment of the RNA with DNAse prior to cDNA synthesis; +DNAse $-\mathrm{RT}=$ treatment with DNAse but no reverse transcription of the RNA; -DNAse - RT $=$ no treatment with DNAse and no reverse transcription of the RNA. sion as a molecular marker for the metabolic activity of $P$. brassicae during clubroot disease.

Trehalose produced by the pathogen can only influence the regulation of plant metabolism when it is released into the plant and when the amounts of trehalose accumulating in the plant cells are sufficiently high to be sensed by the plant. Trehalose could be released actively during the plasmodial stage of $P$. brassicae. In Saccharomyces cerevisiae, export of trehalose occurs via a high-affinity proton symporter (Stambuk et al. 1996). This export of trehalose from the cytosol to the external environment is essential for survival during dehydration and during germination (Cuber et al. 1997). Export of trehalose may also play a role in the developmental cycle of $P$. brassicae. Alternatively, trehalose could be released passively during cell death in later stages of clubroot development when resting spores begin to form (Grsic-Rausch et al. 2000; Kobelt et al. 2000; Ludwig-Müller et al. 1999).

In addition to the strong accumulation of trehalose in infected roots and hypocotyls, we found a significant accumulation of trehalose in the upper parts of the plant (Fig. 1), indicating that trehalose may be released from the pathogen. It can, however, not be excluded that the trehalose found in the leaves was a result of infection of the diseased plants by other microorganisms or due to increased synthesis by the plant.

There was no indication that the starch accumulation in the infected Arabidopsis plants was induced by trehalose: In the leaves, the concentration of trehalose was probably not sufficiently high to induce $A p L 3$ expression (Fig. 2) or starch accumulation (Fig. 1). In the roots and hypocotyls, starch accumulation started earlier (between 14 and 23 days after inoculation; data not shown) than trehalose accumulation (only detectable after 38 days), indicating that trehalose was not involved in the induction of starch formation. In addition, expression of the ADPglucose pyrophosphorylase gene, $A p L 3$, was not induced in the roots and hypocotyls of infected plants (Fig. 2). ApL3 is only inducible by trehalose in the shoots of Arabidopsis plants, but not in the roots (T. Fritzius, unpublished data); therefore, trehalose accumulation in roots cells probably does not affect starch synthesis. Instead, the early accumulation of host carbohydrates, especially of starch, may be due to high cytokinin concentrations, creating a metabolic sink in infected roots (Dekhuijzen 1980). It has been shown that isolated plasmodia of $P$. brassicae are able to synthesize cytokinins from a labeled precursor, ${ }^{14} \mathrm{C}$-adenine (Müller and Hilgenberg 1986). Cytokinin accumulation can increase the starch content in plants by increasing the retention of carbohydrates, accelerating plastid development, and enhancing starch synthesis within the plastids (Miyazawa et al. 1999; Ronzhina and Mokronosov 1994).

The enhanced trehalase activity in clubroot-diseased Arabidopsis plants (Fig. 6) was accompanied by an induction of

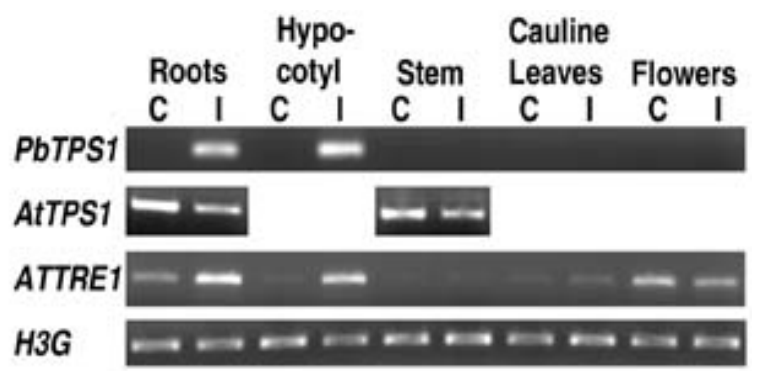

Fig. 5. Reverse transcription-polymerase chain reaction analysis of the expression of the Plasmodiophora brassicae trehalose-6-phosphate synthase (TPS) gene PbTPS1; the Arabidopsis TPS gene AtTPS1; the Arabidopsis trehalase gene ATTRE1; and the Arabidopsis histone gene $H 3 G$ in roots, hypocotyls, stems, and cauline leaves 38 days after inoculation with P. brassicae $; \mathrm{C}=$ control plants and $\mathrm{I}=$ infected plants. 
ATTRE1 expression (Fig. 5). ATTRE1, the only Arabidopsis gene with homology to trehalase genes, has been shown to encode a functional trehalase (Müller et al. 2001); therefore, it is likely that increased expression of ATTRE1 was, at least partially, responsible for the enhanced trehalase activity. In addition, post-transcriptional activation or the presence of a $P$. brassicae trehalase may have contributed to the strong increase in trehalase activity during clubroot disease. The high trehalase activity in the infected plants probably prevents a stronger accumulation of trehalose in the leaves, where it would otherwise interfere with carbohydrate metabolism. ATTRE1 probably is not induced by its substrate trehalose because trehalase activity increases before the accumulation of trehalose. It is more likely that it is regulated by auxin. As shown previously, treatment of the roots of soybean plants with trehalose had no effect on trehalase activity, whereas trehalase activity was strongly increased in auxin-treated roots (Müller et al. 1995b). Synthesis of auxin has been shown to increase during clubroot development (Grsic et al. 1999; Ludwig-Müller et al. 1993, 1996).

In conclusion, we have shown in this study that an increase in trehalose occurs in A. thaliana during clubroot development. We believe that this increase is most probably due to the synthesis of trehalose by the pathogen and not by the host plant, although additional experiments will be necessary to prove this idea. It is possible that an auxin-induced increase in plant trehalase activity prevents an overaccumulation of trehalose in Arabidopsis plants during clubroot disease. We now plan further studies with Arabidopsis mutants affected in trehalose metabolism to examine to what extent trehalose accumulation in plant-pathogen interactions can interfere with plant metabolism and growth.

\section{MATERIALS AND METHODS}

\section{Growth conditions and infection procedure.}

Arabidopsis (ecotype Col-0) seeds were sown onto a mixture of compost, sand, and vermiculite (volume ratio $3: 1: 1$ ). After cold-treatment for 3 to 4 days at $4^{\circ} \mathrm{C}$, the plants were grown in a greenhouse at $20^{\circ} \mathrm{C}$ with additional lighting for 15 $\mathrm{h}$ per day. Fourteen days after transfer to the greenhouse, the plants were inoculated with a suspension of resting spores of a field isolate of Plasmodiophora brassicae. The spores were isolated from infected Chinese cabbage plants (Brassica rapa subsp. pekinensis) as described by Mithen and Magrath (1992). A 5-ml suspension containing spores at $10^{6} \mathrm{ml}^{-1}$ was pipetted around the stem of each plant. The plants were harvested 14, 23, and 38 days after inoculation by separating roots, hypocotyls, stems, rosette leaves, cauline leaves, and flowers. The different tissues were washed in deionized water and frozen in liquid nitrogen.

\section{Determination of carbohydrate contents.}

For the determination of carbohydrate contents, the plant material was homogenized in $80 \%$ ethanol at $80^{\circ} \mathrm{C}$. Resting spores of $P$. brassicae were ground in liquid nitrogen before $80 \%$ ethanol was added. The resulting slurry was vacuum dried, and soluble carbohydrates were extracted and derivatized as described by Müller and associates (1995b) and analyzed by capillary gas chromatography. The gas chromatograph (Carlo Erba Mega 3500; Brechbühler, Zürich, Switzerland) was equipped with a glass column (Capillary JW, $30 \mathrm{~m}$ by $0.323 \mathrm{~mm}$, coated with DB-17; Brechbühler) and with a flame ionization detector. After injection of the derivatized samples, the column was kept for $2 \mathrm{~min}$ at $70^{\circ} \mathrm{C}$, then heated at a rate of $25^{\circ} \mathrm{C}$ per min until a temperature of $170^{\circ} \mathrm{C}$ was reached, followed by a rate of $7^{\circ} \mathrm{C}$ per min to $300^{\circ} \mathrm{C}$ and $5 \mathrm{~min}$ at $300^{\circ} \mathrm{C}$. For the quantification of starch, the starch remaining in the insoluble pellets after the extraction of soluble sugars was digested and measured as described by Wingler and associates (2000).

\section{Determination of trehalase activity.}

The plant material was homogenized in two volumes of a buffer containing 0.1 M MES/KOH (pH 6.3), 1 mM EDTA, 1 $\mathrm{mM}$ phenylmethylsulphonyl fluoride, $1 \mathrm{mM}$ DTT, $0.01 \%$ (vol/vol) Triton-X-100, and $1 \%$ (wt/vol) insoluble polyvinylpyrrolidone (Polyclar AT). After desalting on Bio-Gel P-10 (Bio-Rad Laboratories, Hercules, CA, U.S.A.) equilibrated with $20 \mathrm{mM} \mathrm{MES} / \mathrm{KOH}(\mathrm{pH} 6.3$ ), the extracts were freeze dried. The freeze-dried proteins were dissolved in water, and the trehalase assay was immediately started by addition of 10 $\mathrm{mM}$ trehalose or water (background). After $60 \mathrm{~min}$ at $37^{\circ} \mathrm{C}$, the reaction was stopped by boiling for $2 \mathrm{~min}$. The amount of glucose released by trehalase was measured by HPAEC-PED analysis using an anion-exchange column (CarboPac PA-10, Dionex, Olten, Switzerland) and a pulsed amperometric detector (Dionex) as described by De Virgilio and associates (1993).



Fig. 6. Trehalase activity in the roots plus A, hypocotyls, B, stems, and C, leaves of control plants (open symbols) and of plants infected with Plasmodiophora brassicae (closed symbols). On days 14 and 23, rosette leaves were analyzed; on day 38 , cauline leaves were analyzed. Data are means of between two (control roots, day 14) and four plants \pm standard error. 
The protein concentration in the extracts was determined according to Bradford (1976).

\section{RT-PCR.}

Total RNA was extracted using the Qiagen RNeasy kit (Qiagen, Basel, Switzerland) and treated with DNAse (MessageClean kit; GenHunter, Nashville, TN, U.S.A.). RNA $(1 \mu \mathrm{g})$ was reverse transcribed using a reverse transcription kit (Boehringer, Mannheim, Germany) with both random as well as oligo(dT) primers. The primer pairs were designed to span at least one intron, so that amplification products resulting from potential contamination with genomic DNA would have been separated from the signals for the mRNA transcripts. After the DNAse treatment, no genomic DNA contamination was detectable. For the analysis of gene expression, the following primer pairs were used: ApS (accession number X73365) 5'GATGTAATGCTAGACTTACTAC-3' and 5'-GTCAGTAACATCAGCATCAAG-3'; ApL1 (accession number X73367) 5' TCTATGTGAATGCTTATCTCTC-3' and 5'-CTATGCTCAATCAAGCAGTTGG-3'; ApL2 (accession number X73366) 5' TTCTAAGGTCAAGTTATCCTAC-3' and 5'-TCCTGAAGCTCTACTCCAGAC-3'; ApL3 (accession number X73364) 5' ATGTTCAAGGATACATCTACAG-3' and 5'-CTGAAGCTCAACACCATAGTCA-3'; ACT2 (accession number U41998) 5'-GGAAGGATCTGTACGGTAAC-3' and 5'-TGTGAACGATTCCTGGACCT-3'; ATTRE1 (accession number AC002343) 5'-GAGGAAAGCCAGTAATCCAG-3' and 5'-GTCTCTGACTCAGTAAGAGAG-3'; AtTPS1 (accession number Y08568) 5'-CTCTGTTTCACTACCTTGGACTTCCG-3' and 5'-CTTTGATCATGTCAAGACGATCAACAC-3'; H3G (accession number X60429) 5'-AACCACTGGAGGAGTCAAGA-3' and 5'-CAATTAAGCACGTTCTCCTCTG-3'; PbTPS1 5'-CATGCTTCTGCCGGGCATACT- $3^{\prime}$ and $5^{\prime}$-TTGCCAAGCTGGCCGCCATT- $3^{\prime}$. The infected roots and hypocotyls contained unknown quantities of both $A$. thaliana and $P$. brassicae mRNA; therefore, the amount of cDNA used in the PCR reactions was standardized based on the signal obtained for the constitutive histone gene $(H 3 G)$. The products were separated on agarose gels and visualized by staining with ethidium bromide. The number of cycles in PCR reactions were: 28 cycles for $H 3 G$, 29 cycles for $A p L 3,30$ cycles for $A p S, 32$ cycles for $A p L 1$ and ApL2, 33 cycles for ATTRE1 and AtTPS1, and 34 cycles for PbTPS1. For PbTPS1, strong signals already were obtained at lower cycle numbers, but 34 cycles were used to demonstrate that no expression of PbTPS1 could be detected in noninfected plants or in the leaves, stems, and flowers of infected plants.

\section{Isolation of a TPS gene fragment from $P$. brassicae.}

Degenerate primers were designed for regions of TPS amino acid sequences that are identical for Aspergillus nidulans, Aspergillus niger, Candida albicans, Saccharomyces cerevisiae, and Yarrowia lipolytica, but different for $A$. thaliana. The primers were 5'-TGGGTNCAYGAYTAYCAYYT-3' and '5'-TCDATNCCDATNGGRAANGCTCC-3' $(\mathrm{D}=\mathrm{A}, \mathrm{G}$ or $\mathrm{T} ; \mathrm{N}=\mathrm{A}, \mathrm{C}, \mathrm{G}$ or $\mathrm{T} ; \mathrm{R}=\mathrm{A}$ or $\mathrm{G} ; \mathrm{Y}=\mathrm{C}$ or $\mathrm{T})$. Total RNA was isolated from infected roots and RT-PCR was performed as described above. After separation of the PCR products on an agarose gel, the DNA fragment of the correct size was cleaned using a Geneclean II kit (BIO 101, Vista, CA, U.S.A.) and cloned into the pGEM-T Easy vector (Promega Corp., Madison, WI, U.S.A.). The gene fragment was sequenced in both directions using an ABI PRISM Big Dye Terminator Cycle Sequencing kit and an ABI PRISM 320 Genetic Analyzer (PE Applied Biosystems, Foster City, CA, U.S.A.). The ProDom protein domain database (INRA) was used to search for homology to protein domain families, and ClustalW (EMBL-European Bioinformatics Institute,
Cambridge, U.K.) was used to align the $P$. brassicae sequence with homologous protein sequences.

To confirm that the cloned fragment originated from $P$. bras sicae, genomic DNA was isolated from resting spores of the pathogen as previously described (Grsic-Rausch et al. 2000). Briefly, resting spores of $P$. brassicae were isolated from $100 \mathrm{~g}$ of infected Chinese cabbage root material which was homogenized in $20 \mathrm{mM} \mathrm{NaCl}$ for $5 \mathrm{~min}$ in a Waring Blendor at RT. The homogenate was filtered and the filtrate pressed through an $80-\mu \mathrm{m}$ filter. The filtrate was centrifuged for $5 \mathrm{~min}$ at $30 \times$ $g$, the supernatant filtered through a $20-\mu \mathrm{m}$ filter, and the filtrate again centrifuged for $15 \mathrm{~min}$ at $450 \times g$. The pellet was resuspended in $1 \mathrm{ml}$ of DNAse buffer and incubated for $1 \mathrm{~h}$ at $37^{\circ} \mathrm{C}$ with 10 units DNAseI. After $1 \mathrm{~h}$, the reaction was stopped by the addition of $10 \mathrm{mg}$ of proteinase $\mathrm{K}$ and further incubated at $37^{\circ} \mathrm{C}$ for $2 \mathrm{~h}$. Following incubation, the spores were centrifuged for $10 \mathrm{~min}$ at $20,000 \times g$, and the pellet containing the DNAse/proteinase-treated spores was homogenized in a mortar with sea sand and liquid nitrogen to a very fine powder. The powder then was used for the extraction of genomic DNA according to Murray and Thompson (1980).

Based on the nucleotide sequence of the putative P. brassicae TPS cDNA fragment isolated from infected roots, we designed specific primers and used these primers to amplify a corresponding DNA fragment from genomic DNA of resting spores. The primers were 5 '-AGATCGGGTTCTTCCTGCACACGCC-3' and 5'-CGCCATTCGGTGGGTTGTCCATACC$3^{\prime}$. Twenty-microliter PCRs were performed containing $1 \times \mathrm{Pfx}$ amplification buffer, $0.3 \mathrm{mM}$ of each dNTP, $1 \mathrm{mM} \mathrm{MgSO}_{4}, 0.5$ pmol of each primer, 3x PCRx Enhancer Solution, 1 Unit Pfx (both Life Technologies, Paisley, U.K.), and $500 \mathrm{ng}$ of template. PCR was performed according to standard procedures, using the following program: initial denaturation at $96^{\circ} \mathrm{C}$ for 5 min, followed by 40 cycles of $96^{\circ} \mathrm{C}$ for $45 \mathrm{~s}, 45^{\circ} \mathrm{C}$ for $45 \mathrm{~s}$, and $72^{\circ} \mathrm{C}$ for $45 \mathrm{~s}$ in a Stratagene Robocycler (Stratagene Europe, Amsterdam, The Netherlands). The PCR products were separated on a $2 \%$ agarose gel, stained with ethidium bromide, and purified using the Stratagene gel slice kit (Stratagene Europe). Direct sequencing in both directions was performed on two independently amplified fragments by AGOWA GmbH Sequencing Service (Berlin, Germany). No differences were found in the sequences of the two independently amplified PCR products (data not shown).

\section{LITERATURE CITED}

Agrios, G. N. 1997. Plant Pathology. Academic Press, San Diego, CA, U.S.A.

Blázquez, M. A., Santos, E., Flores, C.-L., Martínez-Zapater, J. M., Salinas, J., and Gancedo, C. 1998. Isolation and molecular characterization of the Arabidopsis TPS1 gene, encoding trehalose-6-phosphate synthase. Plant J. 13:685-689.

Bradford, M. M. 1976. A rapid and sensitive method for the quantitation of microgram quantities of protein utilizing the principle of protein-dye binding. Anal. Biochem. 72:248-254.

Cuber, R, Eleutherio, E. C. A., Pereira, M. D., and Panek, A. D. 1997. The role of the trehalose transporter during germination. Biochim. Biophys. Acta 1330:165-171.

Dekhuijzen, H. M. 1980. The occurrence of free and bound cytokinins in clubroots and Plasmodiophora brassicae infected turnip tissue cultures. Physiol. Plant. 49:169-176.

Dekhuijzen, H. M. 1981. The occurrence of free and bound cytokinins in plasmodia of Plasmodiophora brassicae isolated from tissue cultures of clubroots. Plant Cell Rep. 1:18-20.

Dekhuijzen, H. M., and Overeem, J. C. 1971. The role of cytokinins in clubroot formation. Physiol. Plant Pathol. 1:151-161.

De Virgilio, C., Bürckert, N., Bell, W., Jenö, P., Boller, T., and Wiemken, A. 1993. Disruption of TPS2, the gene encoding the 100-kDa subunit of the trehalose-6-phosphate synthase/phosphatase complex in Saccharomyces cerevisiae, causes accumulation of trehalose-6-phosphate and loss of trehalose-6-phosphate phosphatase activity. Eur. J. Biochem. 
212:315-323.

Eastmond, P. J., van Dijken, A. J. H., Spielman, M., Kerr, A., Tissier, A. F., Dickinson, H. G., Jones, J. D. G., Smeekens, S. C., and Graham, I. A. 2002. Trehalose-6-phosphate synthase 1, which catalyses the first step in trehalose synthesis, is essential for Arabidopsis embryo maturation. Plant J. 29:225-235.

Evans, J. L., and Scholes, J. D. 1995. How does clubroot alter the regulation of carbon metabolism in its host? Asp. Appl. Biol. 42:125-132.

Fritzius, T., Aeschbacher, R., Wiemken, A., and Wingler, A. 2001. Induction of ApL3 expression by trehalose complements the starch-deficient Arabidopsis mutant adg2-1 lacking ApL1, the large subunit of ADPglucose pyrophosphorylase. Plant Physiol. 126:883-889.

Goddijn, O., and Smeekens, S. 1998. Sensing trehalose biosynthesis in plants. Plant J. 14:143-146.

Grsic, S., Kirchheim, B., Pieper, K., Fritsch, M., Hilgenberg, W., and Ludwig-Müller, J. 1999. Auxin biosynthesis in clubroot diseased Chinese cabbage plants and induction by jasmonic acid. Physiol. Plant. 105:521-531.

Grsic-Rausch, S., Kobelt, P., Siemens, J., Bischoff, M., and LudwigMüller, J. 2000. Expression and localization of nitrilase during symptom development of the clubroot disease in Arabidopsis thaliana. Plant Physiol. 122:369-378.

Ingram, D. S., and Tommerup, I. C. 1972. The life history of Plasmodiophora brassicae Woron. Proc. R. Soc. Lond. B 180:103-112.

Ito, S., Ichinose, H., Yanagi, C., Tanaka, S., Kameya-Iwaki, M., and Kishi, F. 1999. Identification of an in planta-induced mRNA of Plasmodiophora brassicae. J. Phytopathol. 147:79-82.

Keen, N. T., and Williams, P. H. 1969. Translocation of sugars into infected cabbage tissues during clubroot development. Plant Physiol. 44:748-754.

Kobelt, P., Siemens, J., and Sacristan, M. D. 2000. Histological characterization of the incompatible interaction between Arabidopsis thaliana and the obligate biotrophic pathogen Plasmodiophora brassicae. Mycol. Res. 104:220-225.

Koch, E., Cox, R., and Williams, P. E. 1991. Infection of Arabidopsis thaliana by Plasmodiophora brassicae. J. Phytopathol. 132:99-104.

Ludwig-Müller, J. 1999. Plasmodiophora brassicae, the causal agent of clubroot disease: A review on molecular and biochemical events in pathogenesis. Z. Pflanzenkr. Pflanzenschutz 106:109-127.

Ludwig-Müller, J., Bendel, U., Thermann, P., Ruppel, M., Epstein, E., and Hilgenberg, W. 1993. Concentration of indole-3-acetic acid in plants of tolerant and susceptible varieties of Chinese cabbage infected with Plasmodiophora brassicae Woron. New Phytol. 125:763-769.

Ludwig-Müller, J., Epstein, E., and Hilgenberg, W. 1996. Auxin-conjugate hydrolysis in Chinese cabbage: Characterization of an amidohydrolase and its role during clubroot disease. Physiol. Plant. 97:627-634.

Ludwig-Müller, J., Ihmig, S., Bennett, R., Kiddle, G., Ruppel, M., and Hilgenberg, W. 1999. The host range of Plasmodiophora brassicae and its relationship to endogenous glucosinolate content. New Phytol. 141:443-458.

Margulis, L., Corliss, J. O., Melkonian, M., and Chapman, D. J. 1989. Handbook of Protoctista. Jones and Partlett Publishers, Boston.

Mithen, R., and Magrath, R. 1992. A contribution to the life history of Plasmodiophora brassicae: secondary plasmodia development in root galls of Arabidopsis thaliana. Mycol. Res. 96:877-885.
Miyazawa, Y., Sakai, A., Miyagishima, S., Takano, H., Kawano, S., and Kuroiwa, T. 1999. Auxin and cytokinin have opposite effects on amyloplast development and the expression of starch synthesis genes in cultured bright yellow-2 tobacco cells. Plant Physiol. 121:461-469.

Müller, J., Aeschbacher, R. A., Sprenger, N., Boller, T., and Wiemken, A. 2000. Disaccharide-mediated regulation of sucrose:fructan-6-fructosyltransferase, a key enzyme of fructan synthesis in barley leaves. Plant Physiol. 123:265-273.

Müller, J., Aeschbacher, R. A., Wingler, A., Boller, T., and Wiemken, A. 2001. Trehalose and trehalase in Arabidopsis thaliana. Plant Physiol 125:1086-1093.

Müller, J., Boller, T., and Wiemken, A. 1995a. Trehalose and trehalase in plants: recent developments. Plant Sci. 112:1-9.

Müller, J., Boller, T., and Wiemken A. 1995b. Effects of validamycin A, a potent trehalase inhibitor, and phytohormones on trehalose metabolism in roots and root nodules of soybean and cowpea. Planta 197:362-368.

Müller, J., Boller, T., and Wiemken, A. 1998. Trehalose affects sucrose synthase and invertase activities in soybean (Glycine max [L.] Merr.) roots. J. Plant Physiol. 153:255-257.

Müller, J., Wiemken, A., and Aeschbacher, R. 1999. Trehalose metabolism in sugar sensing and plant development. Plant Sci. 147:37-47.

Müller, J., Xie, Z.-P., Staehelin, C., Mellor, R. B., Boller, T., and Wiemken, A. 1994. Trehalose and trehalase in root nodules from various legumes. Physiol. Plant. 90:86-92.

Müller, P., and Hilgenberg, W. 1986. Isomers of zeatin and zeatin riboside in clubroot tissue: Evidence for trans-zeatin biosynthesis by Plasmodiophora brassicae. Physiol. Plant. 66:245-250.

Murray, M. G., and Thompson, W. F. 1980. Rapid isolation of high molecular weight plant DNA. Nucleic Acids Res. 8:4321-4325.

Ronzhina, E. S., and Mokronosov, A. T. 1994. Source-sink relations and the role of cytokinins in the regulation of transport and partitioning of organic substances in plants. Russ. J. Plant Physiol. 41:396-406.

Schubert, A., and Wyss, P. 1995. Trehalase activity in mycorrhizal and nonmycorrhizal roots of leek and soybean. Mycorrhiza 5:401-404.

Stambuk, B. U., deAraujo, P. S., Panek, A. D., and Serrano, R. 1996. Kinetics and energetics of trehalose transport in Saccharomyces cerevisiae. Eur. J. Biochem. 237:876-881.

Vogel, G., Aeschbacher, R. A., Müller, J., Boller, T., and Wiemken, A. 1998. Trehalose-6-phosphate phosphatases from Arabidopsis thaliana: Identification by functional complementation of the yeast tps 2 mutant. Plant J. 13:673-683.

Vogel, G., Fiehn, O., Jean-Richard-dit-Bressel, L., Boller T., Wiemken A. Aeschbacher R. A., and Wingler A. 2001. Trehalose metabolism in Arabidopsis: Occurrence of trehalose and molecular cloning and characterization of trehalose-6-phosphate synthase homologues. J. Exp. Bot. 52:1817-1826.

Wagner, W., Wiemken, A., and Matile, P. 1986. Regulation of fructan metabolism in leaves of barley (Hordeum vulgare L. cv. Gerbel). Plant Physiol. 81:444-447.

Williams, P. H., Keen, N. T., Strandberg, J. O., and McNabola, S. S. 1968. Metabolite synthesis and degradation during clubroot development in cabbage hypocotyls. Phytopathology 58:921-928.

Wingler, A., Fritzius, T., Wiemken, A., Boller, T., and Aeschbacher, R. A. 2000. Trehalose induces the ADP-glucose pyrophosphorylase gene, ApL3, and starch synthesis in Arabidopsis. Plant Physiol. 124:105-114. 